\title{
Tuning of PID Controllers based on simplified single parameter optimization
}

\author{
Roberto Sanchis* Julio A. Romero and Pedro Balaguer \\ Departament d'Enginyeria de Sistemes Industrials i Disseny, Universitat Jaume I, Castelló, Spain.
}

\begin{abstract}
A simple method to design PID controllers in the frequency domain based on a simplified constrained optimization is proposed. The method is based on the use of a single tuning parameter, defined as the quotient between the final crossover frequency and the zero of the controller. The tuning procedure is based on the maximization of the controller gain subject to an equality constraint in the phase margin and an inequality constraint in the gain margin. The main advantage of the proposed method is that, even though the maximization of the controller gain is straightforward, since there is only one parameter to be tuned, the solution is close to the optimal tuning obtained with direct numerical optimization methods. Moreover the method is applicable to any linear model structure, including dead time and non-minimum phase systems.
\end{abstract}

Keywords: PID, tuning, frequency domain design, gain margin, phase margin, integral gain maximization.

\section{Introduction}

One of the most prolific research areas in automatic control is devoted to the development of tuning methods for PID controllers. A large number of books and papers have been published about this subject. Some of them are more academic, and deal with a theoretic approach. Other ones are more practical and try to give simple tuning methods that can be easily applied at plant floor by engineers that do not necessarily have a deep background on control theory.

Among the methods for tuning PID controllers, some of the papers found in the literature propose a direct numerical optimization of a given performance index (usually the load IAE, ISE or IE) restricted to some robustness conditions. Among these works, one can cite (Liu and Daley 1999, Hwang and Hsiao 2002, Toscano 2005, Astrom et al. 1998, Panagopoulos et al. 2002). The drawback of this approach is that the numerical optimization tends to be very complex (several local minima could exist).

Another general approach, that can be found for example in (Ho et al. 1998, Madhuranthakam et al. 2008, Toscano 2005, Tavakoli et al. 2005, Astrom and Hagglund 2004), is the approximation of the process to be controlled by a simple first order with time delay or second order with time delay model, and an approximate optimization of a given index, based on those simple models. The final result is usually a set of simple tuning equations, based on a step response of the process, but the drawback is that the behavior of the process must be approximated by those simple models, and this is not always accurate and can lead to a wrong performance.

In this paper, a simple procedure to design PID controllers through minimizing the load IE (maximizing the integral gain), subject to an exact desired phase margin, and a minimum required gain margin constraints, is presented, which in part overcome the drawbacks of the previous approaches. The advantage with respect the direct numerical optimization approaches are the much simpler calculations because the optimization depends on just one single tuning

*Corresponding author. Email: rsanchis@esid.uji.es 
parameter $a$, the quotient between the final crossover frequency and the zero of the controller, while the drawback is that the performance only approximates that of the full optimization approaches. The advantage with respect the approximated model approaches is that a better performance can be obtained because the frequency response data on which the procedure is based is not limited by any particular model structure.

There are some previous works also based on the maximization of the integral gain, but they propose to constrain the optimization to a given value of the maximum of the sensitivity function, and not to a given phase or gain margin. Among these, in (Astrom et al. 1998, Panagopoulos et al. 2002) a direct numerical optimization is proposed, while in (Astrom and Hagglund 2004) an approximation of the process to a FOPDT model is proposed to derive simple tuning rules. The advantages of the proposed approach with respect those ones has been described in the previous paragraph.

On the other hand there are many previous works based on guaranteing a phase and/or gain margin, but none of them (to the author knowledge) proposes to maximize the integral gain. Some of these works propose to use approximate simple process models, as (Ho et al. 1995) and (Ho et al. 1998), where the process is approximated by a FOPDT model. In the first one, the approximate tuning equations for a PI are obtained by imposing predetermined exact phase and gain margins, without taking into account any optimization. In the second one, the approximate tuning equations are derived by minimizing numerically the load ISE, but constrained to exact predefined values of phase and gain margins. One important problem of the approach (besides the error due to the approximation of the process by a very simple FOPDT model), is that forcing an exact prescribed phase margin and also an exact prescribed gain margin may lead to a very restrictive solution, far from the best achievable performance. This is especially important on systems of low order and low delay, where fixing only the prescribed phase margin and maximizing the controller gain leads to a solution where the resulting gain margin is very large. In that case, imposing a predefined exact gain margin (of 3 or 4, for example) would lead to a much worse performance. In (Wang et al. 1999) a tuning procedure is proposed that does not require to approximate the process model, but it also imposes prescribed exact phase and gain margins, plus an arbitrary condition (fixed heuristically) that fixes the closed loop bandwidth as a function of the open loop one, leading to a performance that can be far from the optimum.

The main advantages of the proposed method, with respect the previous works are: i) The optimization is straightforward and can be made by simple hand calculations or with a very low computer cost, since there is only one parameter to be tuned, and it that takes values in a known short interval. The tuning procedure can therefore be implemented in a low cost embedded microprocessor. ii) The performance of the solution is close to the optimal tuning obtained with direct numerical optimization methods that are computationally much more complex, as the ones proposed in (Astrom et al. 1998) and (Panagopoulos et al. 2002). iii) The tuning method is applicable to any linear model order and/or structure, including time delays and non-minimum phase systems. No simple approximated models as FOPDT are required, and the performance exceeds the methods based on those simple approximations. iv) The optimization is constrained to an exact value of the phase margin, but only to a lower bound in the gain margin, avoiding the drawbacks of the methods that fix both exact phase and gain margins. v) The proposed tuning parameter can also be used to obtain a suboptimal performance, for example if the closed loop bandwidth needs to be decreased to avoid high frequency disturbances.

The layout of the paper is as follows: first, the PID design problem is stated. Then, the proposed design methods for PI, PD and PID are described in detail. After that, several simulation design examples carried out over a batch of well known test systems show the validity of the methods (compared with other methods found in the literature). A real experimental design case is then be presented to confirm the practical application of the proposed methods. Finally the conclusions will be summarized. 


\section{Problem statement}

The SISO PID control loop considered in this paper is shown in figure 1, where $r$ is the reference signal to be tracked, $u$ is the control action, $y$ is the controlled output, and $d$ is an input disturbance.

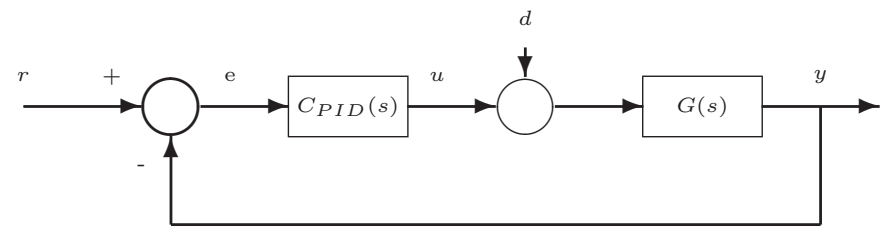

Figure 1. SISO PID control loop

The formulation used for the PI, PD and PID controllers will be as follows. The transfer function of the PI controller is assumed to be

$$
C(s)=K_{p}\left(1+\frac{1}{T_{i} s}\right)=K_{i} \frac{1+\frac{s}{z_{i}}}{s}
$$

where

$$
K_{p}=\frac{K_{i}}{z_{i}} ; T_{i}=\frac{1}{z_{i}}
$$

The transfer function of the PD controller with high frequency filter is assumed to be

$$
C(s)=K_{p}\left(1+\frac{T_{d} s}{1+\frac{T_{d}}{N^{\prime}} s}\right)=K_{p} \frac{1+\frac{s}{z_{d}}}{1+\frac{s}{N z_{d}}}
$$

where

$$
T_{d}=\frac{1}{z_{d}} \frac{N-1}{N} ; \quad N^{\prime}=N-1
$$

Finally, the transfer function of the PID controller with high frequency filter in the derivative term is assumed to be

$$
C(s)=K_{p}\left(1+\frac{T_{d} s}{1+\frac{T_{d} s}{N^{\prime}}}+\frac{1}{T_{i} s}\right)=K_{i} \frac{\left(1+\frac{s}{z_{i}}\right)\left(1+\frac{s}{z_{d}}\right)}{s\left(1+\frac{s}{z_{d} N}\right)}
$$

where the controller zeros have been assumed to be real and

$$
\begin{aligned}
& K_{p}=K_{i}\left(\frac{z_{d}+z_{i}}{z_{d} z_{i}}-\frac{1}{z_{d} N}\right) ; T_{d}=\frac{1}{z_{d}+z_{i}-\frac{z_{i}}{N}}-\frac{1}{z_{d} N} \\
& T_{i}=\frac{z_{d}+z_{i}}{z_{d} z_{i}}-\frac{1}{z_{d} N} ; N^{\prime}=\frac{z_{d} N}{z_{d}+z_{i}-\frac{z_{i}}{N}}-1
\end{aligned}
$$

The process is assumed to be linear, or, if it is non linear, it is assumed to stay in the vicinity of an operating point where it can be approximated by a linear model. A non parametric 
model of the process in that operating point is assumed to be known in the form of a list of numerical frequency response data (frequency, magnitude, phase). This data can be obtained from experiments, by means of an open loop experiment with an exciting input and calculating the ETFE (as described for example in (Ljung 1999)), or by closed loop relay type experiments (as described for example in (Tan et al. 2005)). The objective of the PID controller to be designed is to reach an adequate performance and robustness of the controlled system, expressed in the following design specifications:

- Required phase margin: $\phi_{m, r}$. The phase margin $\left(\phi_{m}\right)$ should be equal to this value $\left(\phi_{m}=\right.$ $\left.\phi_{m, r}\right)$.

- Minimum required gain margin: $\gamma_{m, r}$. The gain margin $\left(\gamma_{m}\right)$ should be larger than or equal to this value $\left(\gamma_{m} \geq \gamma_{m, r}\right)$.

- Good disturbance rejection. The step disturbance IAE or steady state error should be as low as possible.

Other specifications, like position or velocity error, or closed loop bandwidth should guide in the selection of the most appropriate controller (PI, PD or PID), and should be checked after the controller is designed.

The proposed design methods for PI, PD and PID controllers follow the same strategy and, therefore, a unified description of the three controllers will be developed.

\section{$3 \quad$ PID design methods}

Before starting to describe the details of the design method, some previous considerations have to be made.

\subsection{Previous considerations}

First, in the case of the PID controller, the two zeros are imposed to be equal, i.e. $z_{i}=z_{d}$, in order to simplify the design method. In other works, as (Krinstianson and Lennartson 2006a) and (Krinstianson and Lennartson 2006b), this restriction is not applied, and even complex zeros are allowed. This restriction could result in a significant loss of performance for some particular processes (especially those with highly resonant dynamics). However, for the batch of models proposed in (Astrom et al. 1998) and (Panagopoulos et al. 2002), that are representative of most of the industrial processes dynamics, the developed examples (as can be seen in tables 3 and 4) conclude that the loss of performance due to this restriction is not important. In those comparative examples, the proposed approach (with equal and real zeros) is compared with a pure numerical optimization approach (MIGO) where the zeros are independently fixed, leading to complex zeros most of the times.

On the other hand, the proposed approach assumes that the designer has first selected the derivative filter factor $N$, based on the information available about the measurement noise. This filter factor is usually selected in a range between 5 and 20 . If the high frequency measurement noise is low, a large value of $N$ can be selected. On the contrary, if the high frequency measurement noise is important, a low value of $N$ should be chosen. Typical intermediate cases lie around $N=10$. The price paid by an improved high frequency measurement noise filtering, achieved by a low value of $N$, is the lower closed loop bandwidth and higher IAE due to the reduced phase lead of the controller. Therefore the selection of $N$ is a compromise between high frequency robustness and performance. In (Krinstianson and Lennartson 2006a) and (Krinstianson and Lennartson 2006b), this factor is included in the set of parameters to be designed, but this makes the optimization problem much more complex, while the idea of the proposed method is to simplify the design. On the other hand, the exact selection of this parameter is not critical for the final result, in the sense that, for example, a value $N=5$ or $N=6$ lead to very 
similar designs.

\subsection{Detailed design method}

The design method can be summarized as a constrained optimization approach in which the proposed PID parameters are those that maximize the controller gain $\left(K_{i}\right.$ for PI and PID or $K_{p}$ for PD), subject to the following constraints: $\phi_{m}=\phi_{m, r}, \gamma_{m} \geq \gamma_{m, r}, z_{d}=z_{i}$.

Remark 3.1 In the case of PI or PID, the maximization of $K_{i}$ is equivalent to the minimization of the integral of the error (IE) of the step disturbance response, because it is well known that

$$
I E=\lim _{t \rightarrow \infty} \int_{0}^{t} e(\tau) d \tau=\lim _{s \rightarrow 0} \frac{G(s)}{s(1+C(s) G(s))}=\frac{1}{K_{i}}
$$

If the phase and gain margins specifications are large enough, then the closed loop response is not too oscillatory, and the IE is similar to the IAE. Therefore, the approach is equivalent to an approximate minimization of the IAE. In the case of a PD controller, the maximization of $K_{p}$ is equivalent to the minimization of the steady state disturbance error, because of the well known result

$$
\lim _{t \rightarrow \infty} e(t)=\lim _{s \rightarrow 0} \frac{G(s)}{(1+C(s) G(s))}=\frac{G(0)}{1+K_{p} G(0)}
$$

The key to solve the previous constraint maximization in a straightforward way is to introduce a tuning parameter, that is the relation between the final gain crossover frequency of the process with controller and the controller zero

$$
a=\frac{\omega_{c g}}{z_{c}} \quad ; \quad z_{c}=z_{i}, z_{d}
$$

where $\omega_{c g}$ is the frequency where the magnitude of the open loop frequency response of the process plus controller is 1, i.e. where the phase margin is measured.

For a given value of this parameter $a$, the phase of the controller at the final crossover frequency $\omega_{c g}$ depends only on that value. For the different controllers one obtains

$$
\begin{gathered}
P I: \quad \arg \left(C\left(j \omega_{c g}\right)\right)=\arctan (a)-\frac{\pi}{2}=-\arctan \left(\frac{1}{a}\right) \\
P D: \quad \arg \left(C\left(j \omega_{c g}\right)\right)=\arctan (a)-\arctan \left(\frac{a}{N}\right) \\
\text { PID : } \arg \left(C\left(j \omega_{c g}\right)\right)=2 \arctan (a)-\arctan \left(\frac{a}{N}\right)-\frac{\pi}{2}
\end{gathered}
$$

Therefore, for a given value of parameter $a$, the calculation of the controller is automatic, following two steps

(1) The final crossover frequency $\left(\omega_{c g}\right)$ is obtained as the frequency where the phase of the system fulfills the phase margin equation, i.e. where the phase of the system is

$$
\arg \left(G\left(j \omega_{c g}\right)\right)=-\pi+\phi_{m, r}-\arg \left(C\left(j \omega_{c g}\right)\right)
$$


Then, the value of the zero $\left(z_{i}\right.$ or $\left.z_{d}\right)$ is calculated as

$$
z_{c}=\frac{\omega_{c g}}{a}
$$

(2) The value of $K_{i}$ (or $K_{p}$ for $\mathrm{PD}$ ) is calculated from the condition of unit magnitude at the final crossover frequency

$$
\left|C\left(j \omega_{c g}\right) G\left(j \omega_{c g}\right)\right|=1 \quad \rightarrow \quad K_{i}\left(\text { or } K_{p}\right)
$$

The resulting equation for each type of controller is:

$$
\begin{gathered}
P I: \quad K_{i}=\frac{\omega_{c g}}{\left|G\left(j \omega_{c g}\right)\right| \sqrt{1+a^{2}}} \\
P D: \quad K_{p}=\frac{\sqrt{1+\frac{a^{2}}{N^{2}}}}{\left|G\left(j \omega_{c g}\right)\right| \sqrt{1+a^{2}}} \\
\text { PID : } \quad K_{i}=\frac{\omega_{c g} \sqrt{1+\frac{a^{2}}{N^{2}}}}{\left|G\left(j \omega_{c g}\right)\right|\left(1+a^{2}\right)}
\end{gathered}
$$

In the previous steps, the available frequency response data of the process is used, interpolating, if necessary, between the available points. Once the controller parameters are defined, the final gain margin, $\gamma_{m}$, is calculated. Applying the previous steps for different values of $a$, two graphs relating $a$ with $K_{i}$ and $\gamma_{m}$ can be easily built, and the value of $a$ where $K_{i}$ reaches a maximum value, constrained to $\gamma_{m} \geq \gamma_{m, r}$ can be obtained straightforwardly (as shown in figure 2). The usual range of values to be taken into account are from $a=0.1$ till $a=5$, as it will be shown in the examples. Once the values of $K_{i}$ and $z_{c}$ are obtained, the parameters of the controller $\left(K_{p}, T_{d}\right.$ and $\left.T_{i}\right)$ are calculated applying equations (2), (4) or (6).

Some design methods described on well known classical books ((Phillips and Harbor 1999, Dorf and Bishop 2007, Franklin et al. 2005, D'azzo and Houpis 1966, Ogata 2003)) propose to fix the parameter $a$ for PI and PID at values in the range $a=8$ to $a=10$, while for PD controllers they propose to fix the value $a=\sqrt{N}$. However, as it will be shown in the examples, these are not usually good choices. Instead, our proposal is to select the value that maximizes $K_{i}$ (or $K_{p}$ for PD), constrained to $\gamma_{m} \geq \gamma_{m, r}$ and $\phi_{m}=\phi_{m, r}$.

\subsection{Remarks}

Remark 1: The maximization of gain $K_{i}$ is simple, because there is only one parameter $(a)$ to be varied. It can even be done "by hand", because most of the times the optimum value lies between $a=0.5$ and $a=5$, and its exact value is not critical. Of course, to find the optimum value of parameter $a$ numerically is immediate using a computer with a low cost algorithm.

Remark 2: In some cases, the optimum value of $a$ could result in a too high closed loop bandwidth (for example if an important high frequency noise is present and should not be amplified by the closed loop). In those cases, the $a$ parameter can be used as a very simple tuning factor: reducing the value of $a$ from the optimum, results on reducing the closed loop bandwidth, and hence, on increasing the attenuation of the high frequency noise (the cost is, of course, increasing the IAE). 
Remark 3: The proposed design approach does not take into account the use of a weighting factor for the proportional term of the reference, in the case of PI and PID controllers. This weighting factor $b<1$ is usually added in the calculation of the proportional part of the control action as $u_{p}=K_{p}\left(b y_{r e f}-y\right)$. If the overshoot of the step reference response is too high, an adequate selection of this factor $b$ can reduce it to a lower level. Trial and error can be used to adjust the value of $b$. If a transfer function model of the process is available, this trial and error can be made in simulations.

Remark 4: The design method needs to know the frequency response of the plant. This can be obtained by means of an experiment, with a sufficiently exciting input (for example with a chirp input signal). In practical industrial processes, this exciting input signal may be difficult to be applied. In order to overcome this difficulty, and to make more applicable in practice the proposed design method, a simple straight line approximation of the frequency response can be used. For this approximation, only two points of the frequency response are needed. These points can be obtained by two simple relay feedback experiments (as described for example in (Tan et al. 2005)). The two points should be near the $180^{\circ}$ phase, such that an approximation of the straight tangent line of the Bode plot at that phase is obtained. The experimental example shows the applicability of this idea.

\section{Simulation examples}

In this section several examples are used to illustrate the main issues in the design of PI, PD and PID controllers using the methodology proposed on this paper.

In the next examples, a computer has been used in order to obtain a graph that relates the values of parameter $a$ to the resulting $K_{i}$ and gain margin. These graphs show that there is a quite flat region around the local maximum, and hence, the non exact selection of the optimum $a$ value is not critical for the final performance, because the gain $K_{i}$ (and hence the IAE) will be only slightly worse than the optimum.

\subsection{PID design}

Consider a system modelled by the following fourth order transfer function:

$$
G(s)=\frac{15625}{(s+1)(s+5)(s+25)(s+125)}
$$

The specifications for the controller design are assumed to be: $\phi_{m}=50 \mathrm{deg}, \gamma_{m} \geq 2$. The signal to noise ratio on the output measured signal is assumed to be low, so a low value is selected for the filter parameter: $N=5$.

The values of $K_{i}$ and $\gamma_{m}$, for $0.2 \leq a \leq 5$ are shown in the figure 2 . The condition $\gamma_{m} \geq 2$ is fulfilled for all $a$ in the range $[0.2,5]$. The controller with approximate optimal performance in the sense of disturbance IAE is obtained for $a=1.8$ (design A), where $K_{i}$ is maximum.

Two other controllers have been selected to be compared with the optimal one: points $\mathrm{B}$ and $\mathrm{C}$, corresponding to designs with $a=1$ and $a=3$ respectively. The figures 3 and 4 show the closed loop step reference and disturbance response without measurement noise.

Clearly, the best disturbance response is that of controller A. The controller C leads to the largest closed loop bandwidth and the fastest reference response, but its disturbance response is the slowest to converge. On the other hand, controller B leads to the lowest closed loop bandwidth and the slowest reference response. This example shows how the lower the value of $a$, the lower the closed loop bandwidth of the resulting designed control system. The parameter $a$ could then be used to tune the desired closed loop bandwidth, for example to reduce the effect of 


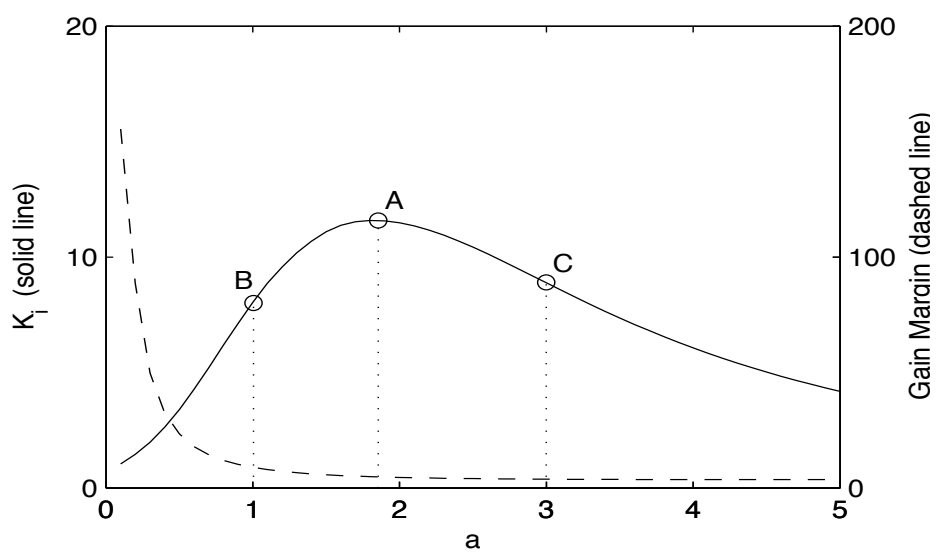

Figure 2. Controller gain $K_{i}$ and Gain Margin as a function of parameter $a$ for PID controller in example 3.1.

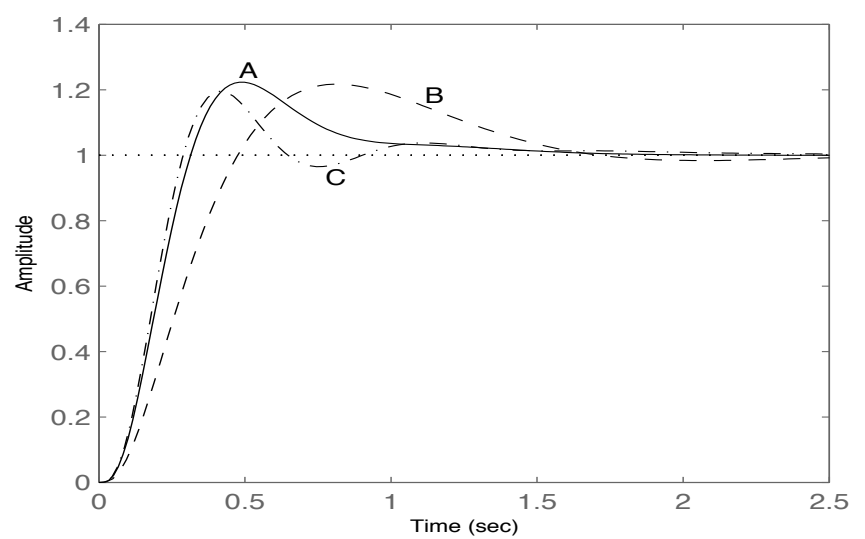

Figure 3. Closed loop response to step change on reference in example 3.1: solid line $(\mathrm{a}=1.8)$; dashed line $(\mathrm{a}=1)$; dasheddotted line $(a=3)$.

high frequency noise in the control system. The figure 5 shows the steady closed loop response of controllers $\mathrm{A}$ and $\mathrm{B}$ when the measurement noise is added to the system output. The controller B achieves a significant noise attenuation (the cost is, of course, a slower response).

The selection of values of $a$ larger than the optimal may lead to a higher final bandwidth, but this should be used with caution, due to the degradation of the disturbance response (and eventually also of the reference response). The main reason is that large values of $a$ lead to low values of integral gain $\left(K_{p} / T_{i}\right)$, resulting in a slow correction of steady state error. This effect is presented in the figures 6 and 7 , where the closed loop response to step reference and disturbance is shown for controllers designed with $a=6, a=8$ and $a=10$.

The effect of the selected filter factor $N$ on the achieved performance is shown in table 1 . The resulting IAE for low values of $N$ is higher than for high $N$ values. This is a result of the lower phase lead of the controller that leads to a lower closed loop bandwidth. Of course, this slower response is the price for an improved filtering of high frequency measurement noise. Nevertheless, the exact value of $N$ is not critical, as a small change in this factor results in a small change in the designed PID, as can be noted for $N=5$ and $N=6$.

\subsection{PI design}

In this example a PI controller is designed for the non-minimum phase system (19). The specifications are: $\phi_{m}=50 \mathrm{deg}, \gamma_{m} \geq 2$. 


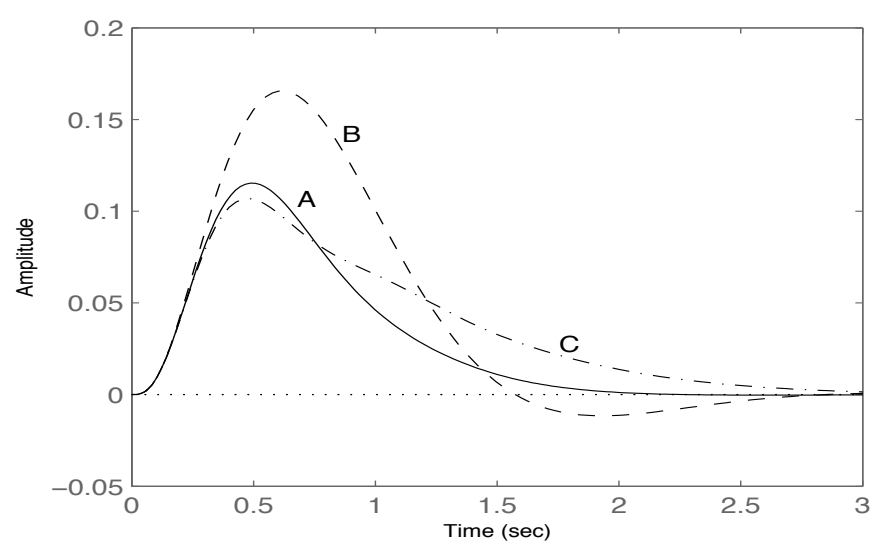

Figure 4. Closed loop response to step change on disturbance in example 3.1: solid line ( $\mathrm{a}=1.8)$; dashed line ( $\mathrm{a}=1$ ); dasheddotted line $(\mathrm{a}=3)$.

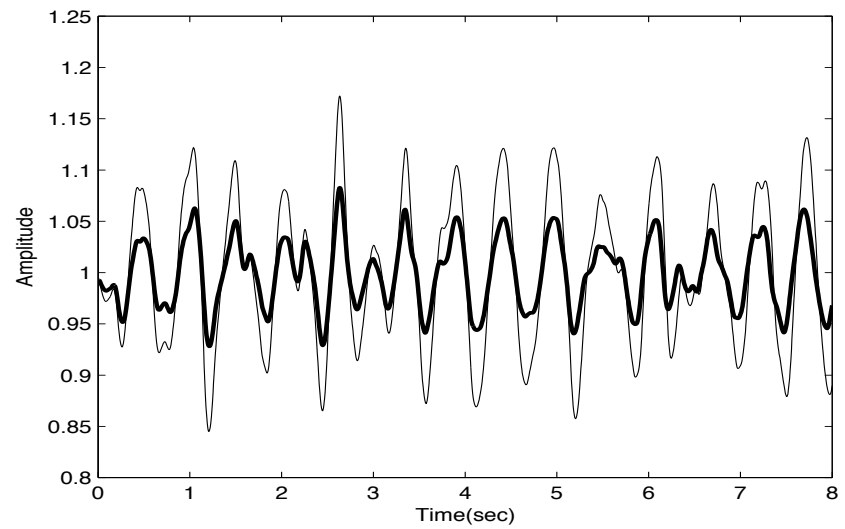

Figure 5. Closed loop steady state response with measurement noise in example 3.1: controller A $a_{\text {opt }}=1.8$ (light line); controller $\mathrm{B} \mathrm{a}=1$ (dark line).

Table 1. Performance of the resulting PID controllers of the proposed design method for different values of $\mathrm{N}$.

\begin{tabular}{ccccccc}
\hline$N$ & $K_{p}$ & $T_{i}$ & $T_{d}$ & $w_{c}$ & $w_{a}$ & IAE \\
\hline 5 & 6.88 & 0.59 & 0.12 & 5.49 & 9.65 & 0.087 \\
6 & 7.87 & 0.59 & 0.12 & 6.13 & 10.00 & 0.075 \\
10 & 12.55 & 0.55 & 0.13 & 8.59 & 13.00 & 0.043 \\
20 & 14.02 & 0.54 & 0.13 & 9.25 & 17.40 & 0.039 \\
\hline
\end{tabular}

$$
G(s)=\frac{-3.5 s+1}{(s+1)^{3}}
$$

The controller gain $K_{i}$ and $\gamma_{m}$ are plotted as a function of $a$ in the figure 8. The maximum of $K_{i}$ is obtained for $a=0.7$ (design B), but the gain margin is too low $\left(\gamma_{m}=1.23<2\right)$. The maximum of $K_{i}$ subject to the constraint of $\gamma_{m} \geq 2$ obviously corresponds to point A, where $a=0.13$, hence this is the proposed design. The closed loop response of controllers A and B is compared in figures 9 and 10, where the excessive oscillation of design B (due to a low gain margin) is shown. 


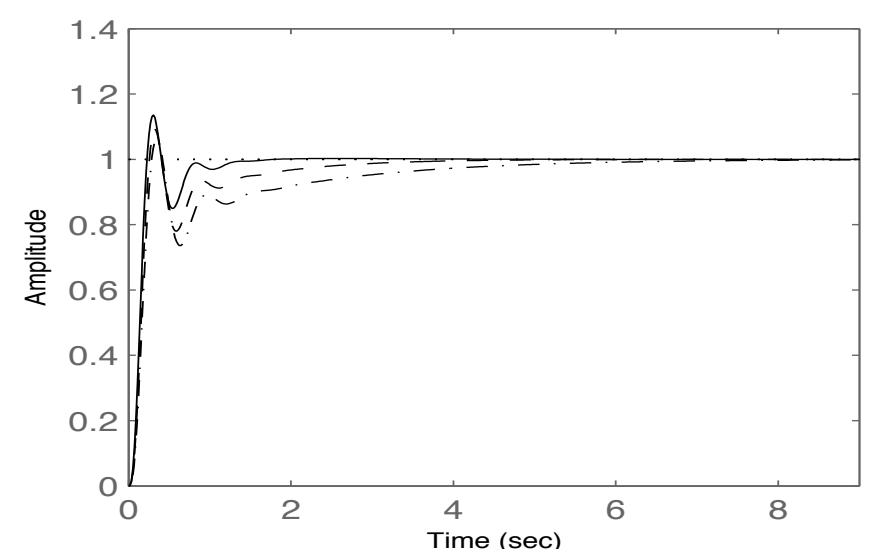

Figure 6. Closed loop response to step change on reference in example 3.1: solid line $(a=6)$; dashed line $(a=8)$; dashed-dotted line $(a=10)$.

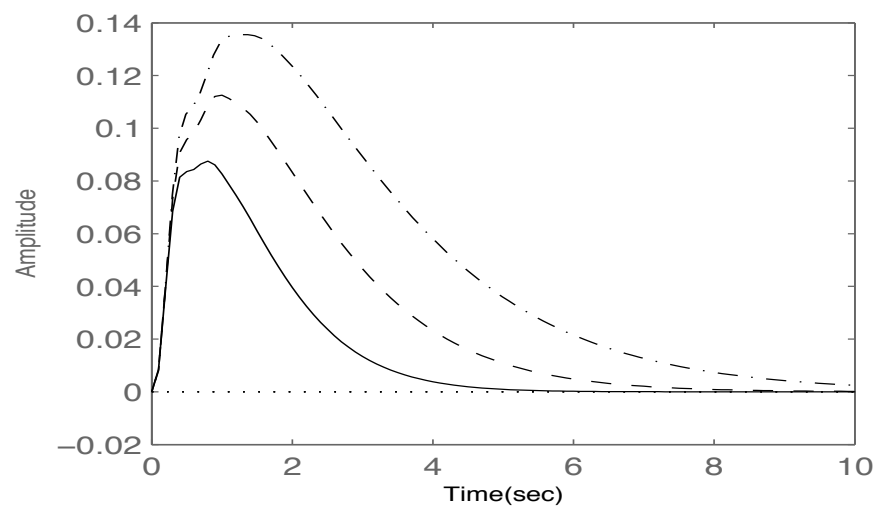

Figure 7. Closed loop response to step change on disturbance in example 3.1: solid line $(a=6)$; dashed line $(a=8)$; dasheddotted line $(a=10)$.

\section{Comparison with other approaches}

The proposed design strategy has been compared with other approaches based on numerical maximization of the integral gain. In (Astrom et al. 1998) and (Panagopoulos et al. 2002), two approaches to design PI and PID controllers respectively are presented. The methods, called MIGO (M-constrained integral gain optimization) are based on a numerical minimization of the disturbance IE, restricted to a constraint in the maximum sensitivity. In (Astrom and Hagglund 2004) the MIGO method was used to find simple tuning rules for PID control called AMIGO (approximate MIGO), based on the approximation of the process by simple FOPDT models.

For the comparison study, the test batch of models proposed in (Astrom et al. 1998) and (Panagopoulos et al. 2002) has been used. In the case of PI controllers the considered models are:

$$
\begin{gathered}
G_{1}(s)=\frac{1}{(s+1)^{3}} \\
G_{2}(s)=\frac{1}{(s+1)(1+0.2 s)\left(1+0.2^{2} s\right)\left(1+0.2^{3} s\right)}
\end{gathered}
$$




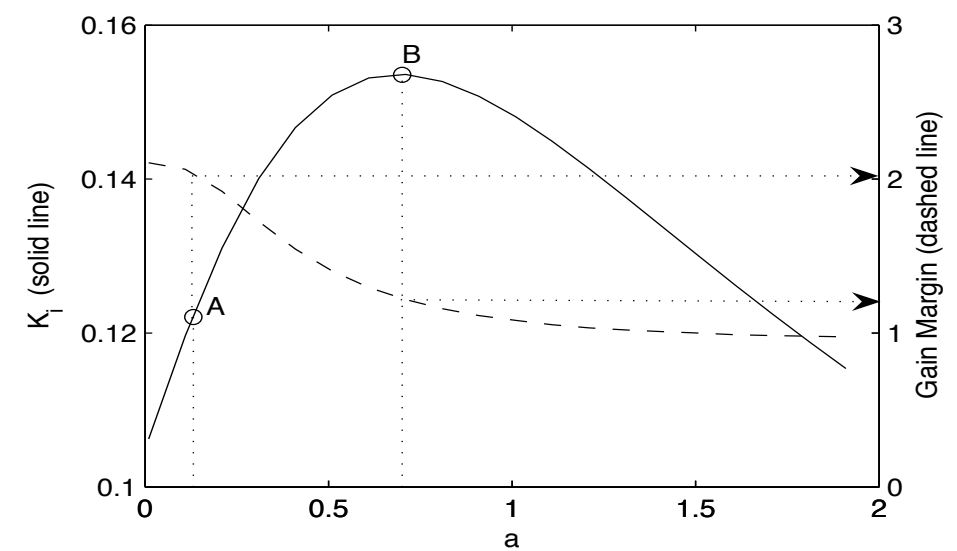

Figure 8. Controller gain $K_{i}$ and Gain Margin as a function of parameter $a$ for PI controller in example 3.2.

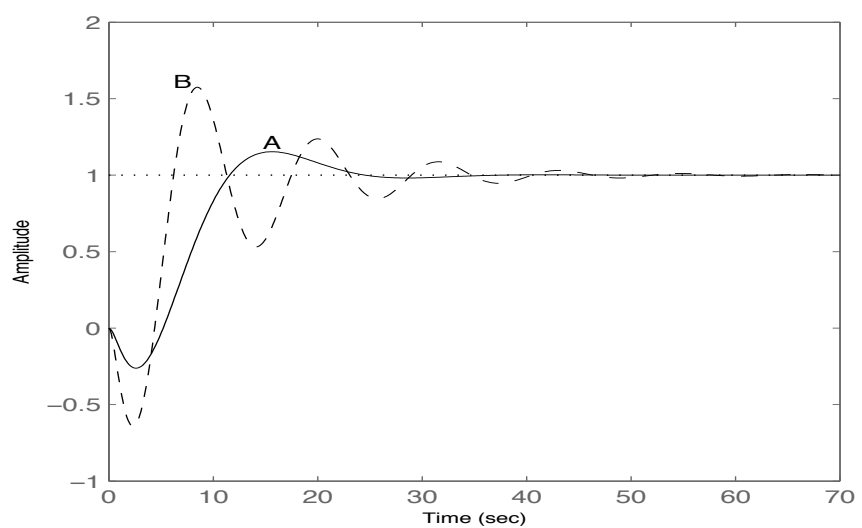

Figure 9. Closed loop response to step change on reference in example 3.2: solid line (design A: a=0.13); dashed line (design $\mathrm{B}: \mathrm{a}=0.7)$.

$$
\begin{gathered}
G_{3}(s)=\frac{e^{-15 s}}{(s+1)^{3}} \\
G_{4}(s)=\frac{1}{s(s+1)^{2}} \\
G_{5}(s)=\frac{1-2 s}{(s+1)^{3}} \\
G_{6}(s)=\frac{9}{(s+1)\left(s^{2}+2 s+9\right)}
\end{gathered}
$$

For the PID controllers the test batch of models is:

$$
G_{1}(s)=\frac{1}{s(s+1)^{3}}
$$




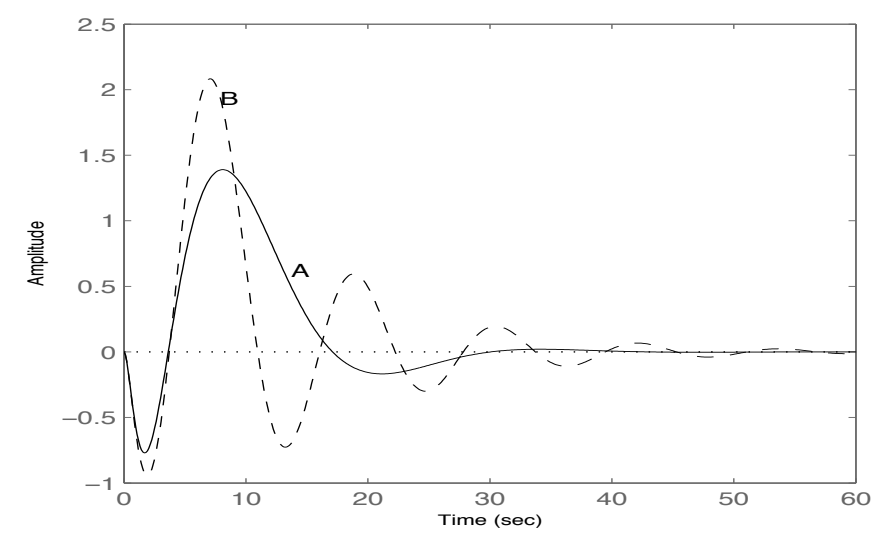

Figure 10. Closed loop response to step change on disturbance in example 3.2: solid line (design A: a=0.13); dashed line (design $\mathrm{B}$ : $\mathrm{a}=0.7)$.

$$
\begin{gathered}
G_{2}(s)=\frac{e^{-5 s}}{(s+1)^{3}} \\
G_{3}(s)=\frac{1}{(s+1)(1+0.2 s)\left(1+0.2^{2} s\right)\left(1+0.2^{3} s\right)} \\
G_{4,5,6,7}(s)=\frac{1}{(s+1)^{\alpha}} \quad \alpha=4,5,6,7 \\
G_{8}(s)=\frac{-2(s-2)}{(s+1)^{3}}
\end{gathered}
$$

These models capture the typical dynamics encountered in control applications: multiple poles, integrator, time delay, non minimum phase.

For these models, PI and PID controllers have been designed following the proposed design method. In (Astrom et al. 1998) and (Panagopoulos et al. 2002), two values of the maximum sensitivity are considered $\left(M_{s}=2\right.$ and $\left.M_{s}=1.4\right)$, representing two different compromises between performance and robustness. In order to compare with these works, two pairs of values of phase and gain margins have been used in the proposed design $\left(\phi_{m, r}=35^{\circ}, \gamma_{m, r}=2\right.$ and $\phi_{m, r}=65^{\circ}, \gamma_{m, r}=3.5$ ) with the same objective. The value of $M_{s}$ is in general a better parameter to select a compromise between performance and robustness, but the $\phi_{m}$ and $\gamma_{m}$ used in the proposed method are also valid for that purpose, and the computation of the resulting design procedure is much simpler.

The table 2 shows the comparative results obtained by the proposed PI design method and the one in (Astrom et al. 1998). The resulting maximum sensitivity, phase margin, gain margin and IAE are shown. In general, the results obtained with the two methods are quite similar, in the sense that for a given system and a given value of $M_{s}$ in the design (Astrom et al. 1998), there are values of $\phi_{m}, \gamma_{m}$ that lead to a similar behavior with the proposed method. Some particular cases should be commented from the table 2 . In models $G_{3}$ and $G_{5}$, the design with $\phi_{m}=35^{\circ}, \gamma_{m} \geq 2$ has no solution (can not reach a $\gamma_{m} \geq 2$ for any $a$ value), therefore, the $\phi_{m}$ had to be increased till there existed a solution for which $\gamma_{m} \geq 2$. On the other hand, in model $G_{4}$, with $\phi_{m}=65$, the resulting $I A E$ is much larger than the one obtained with $M_{s}=1.4$, because a much more robust design is reached. In that case, taking a value of $\phi_{m}=50$ (instead 
of 65$)$ would reach a very similar performance to the one obtained with $M_{s}=1.4$.

\begin{tabular}{|c|c|c|c|c|c|c|}
\hline$G_{n}$ & Design & $M_{s}$ & $\phi_{m}$ & $\gamma_{m}$ & $I A E$ & $I E$ \\
\hline \multirow{4}{*}{$G_{1}$} & $\operatorname{FR}\left(M_{f}=35\right)$ & 2.33 & 35 & 3.24 & 1.56 & 1.21 \\
\hline & $\operatorname{FR}\left(M_{f}=65\right)$ & 1.56 & 65 & 4.73 & 2.47 & 2.47 \\
\hline & $\operatorname{MIGO}\left(M_{s}=2\right)$ & 2 & 42.02 & 3.25 & 1.88 & 1.45 \\
\hline & $\operatorname{MIGO}\left(M_{s}=1.4\right)$ & 1.4 & 67.93 & 6.74 & 3.07 & 3.07 \\
\hline \multirow{4}{*}{$G_{2}$} & $\operatorname{FR}\left(M_{f}=35\right)$ & 2.01 & 35 & 4.49 & 0.14 & 0.14 \\
\hline & $\operatorname{FR}\left(M_{f}=65\right)$ & 1.36 & 65 & 10.9 & 0.50 & 0.50 \\
\hline & $\operatorname{MIGO}\left(M_{s}=2\right)$ & 2 & 35.14 & 4.90 & 0.15 & 0.14 \\
\hline & $\operatorname{MIGO}\left(M_{s}=1.4\right)$ & 1.5 & 56.07 & 11.56 & 0.38 & 0.39 \\
\hline \multirow{4}{*}{$G_{3}$} & $\operatorname{FR}\left(M_{f}=35\right)$ & 2.2 & $45.00^{*}$ & 2.00 & 36.9 & 20.4 \\
\hline & $\operatorname{FR}\left(M_{f}=65\right)$ & 2.06 & 65 & 3.50 & 36.80 & 35.90 \\
\hline & $\operatorname{MIGO}\left(M_{s}=2\right)$ & 2 & 53.93 & 2.11 & 27.73 & 20.8 \\
\hline & $\operatorname{MIGO}\left(M_{s}=1.4\right)$ & 1.4 & 71.62 & 3.77 & 37.50 & 37.5 \\
\hline \multirow{4}{*}{$G_{4}$} & $\operatorname{FR}\left(M_{f}=35\right)$ & 1.99 & 35 & 4.15 & 25.70 & 25.7 \\
\hline & $\operatorname{FR}\left(M_{f}=65\right)$ & 1.25 & 65 & 12.8 & 315.00 & 315 \\
\hline & $\operatorname{MIGO}\left(M_{s}=2\right)$ & 2 & 33.03 & 4.50 & 25.00 & 24.0 \\
\hline & $\operatorname{MIGO}\left(M_{s}=1.4\right)$ & 1.4 & 47.94 & 10.24 & 86.59 & 84.0 \\
\hline \multirow{4}{*}{$G_{5}$} & $\operatorname{FR}\left(M_{f}=35\right)$ & 2.24 & $45.00^{*}$ & 2.00 & 9.03 & 4.95 \\
\hline & $\operatorname{FR}\left(M_{f}=65\right)$ & 2.09 & 65 & 3.50 & 9.85 & 8.75 \\
\hline & $\operatorname{MIGO}\left(M_{s}=2\right)$ & 2 & 52.85 & 2.18 & 7.74 & 5.42 \\
\hline & $\operatorname{MIGO}\left(M_{s}=1.4\right)$ & 1.4 & 71.15 & 3.91 & 11.00 & 9.90 \\
\hline \multirow{4}{*}{$G_{6}$} & $\operatorname{FR}\left(M_{f}=35\right)$ & 2.30 & 35 & 2.00 & 1.22 & 0.59 \\
\hline & $\operatorname{FR}\left(M_{f}=65\right)$ & 2.17 & 65 & 3.50 & 1.29 & 1.21 \\
\hline & $\operatorname{MIGO}\left(M_{s}=2\right)$ & 2 & 42.88 & 2.15 & 1.01 & 0.65 \\
\hline & $\operatorname{MIGO}\left(M_{s}=1.4\right)$ & 1.4 & 59.16 & 3.84 & 1.36 & 1.19 \\
\hline
\end{tabular}

The tables 3 and 4 shows the comparative results obtained by the proposed PID design method and the ones in (Panagopoulos et al. 2002) and (Astrom and Hagglund 2004). The resulting maximum sensitivity, phase margin, gain margin and IAE are shown. In general, the results obtained with the three methods are quite similar, in the sense that for a given system and a given value of $M_{s}$ in the design (Panagopoulos et al. 2002), there are values of $\phi_{m}, \gamma_{m}$ that lead to a similar behavior with the proposed method. However, the approximation implicit in the method (Astrom and Hagglund 2004) leads in some cases to a quite worse performance, as for example in $G_{1}, G_{3}, G_{4}$ and $G_{5}$. Some particular cases should be commented from the tables 3 and 4 . In models $G_{2}$ and $G_{8}$, the design with $\phi_{m}=35^{\circ}, \gamma_{m} \geq 2$ has no solution (can not reach a $\gamma_{m} \geq 2$ for any $a$ value), therefore, the $\phi_{m}$ had to be increased till there existed a solution for which $\gamma_{m} \geq 2$.

\section{Experimental case study}

This section illustrates the applicability of the proposed PID design method to a real experimental case. The process to be controlled is a thermal system, composed by a heater resistor attached to an iron plate inside a plastic cover. A switched amplifier drives the resistor, while a thermocouple with an amplifier is used to measure the temperature. The system is completed by a small fan that can be used to apply a disturbance to the plant. The PID controller is implemented by a PC with a data acquisition card using Matlab Real Time Windows Target software. A simple Euler discretization with period $T=1$ second and a parallel realization of the controller with anti windup is used. A picture of the complete thermal system (without the plastic cover) is presented in the figure 11.

In this case study, unlike the previous examples, the PID controller is designed using a nonparametric model of the system, defined by a numerical list of frequency response data points, estimated by spectral analysis technics. The experimental study included the following steps: System identification (an experiment is carried out on the plant in order to obtain the frequency 
Table 3. Comparison of the results reached by the proposed PID design method and the ones in (Panagopoulos et al. 2002) and (Astrom and Hagglund 2004). FR: Frequency response based method, MIGO: M-constrained integral gain optimization method, AMIGO: approximate MIGO method.

\begin{tabular}{|c|c|c|c|c|c|c|}
\hline$G_{n}$ & Design & $M_{s}$ & $\phi_{m}$ & $\gamma_{m}$ & $I A E$ & $I E$ \\
\hline \multirow{6}{*}{$G_{1}$} & $\operatorname{FR}\left(M_{f}=35\right)$ & 2.17 & 35 & 3.00 & 9.34 & 9.08 \\
\hline & $\operatorname{FR}\left(M_{f}=65\right)$ & 1.53 & 65 & 4.96 & 30.60 & 28.5 \\
\hline & $\operatorname{MIGO}\left(M_{s}=2\right)$ & 2 & 41.69 & 3.12 & 9.58 & 6.61 \\
\hline & $\operatorname{MIGO}\left(M_{s}=1.4\right)$ & 1.4 & 50.52 & 6.43 & 30.45 & 20.4 \\
\hline & $\operatorname{AMIGO}\left(M_{s}=2\right)$ & - & - & - & - & - \\
\hline & $\operatorname{AMIGO}\left(M_{s}=1.4\right)$ & 1.61 & 48.3 & 5.38 & 49.5 & 49.5 \\
\hline \multirow{6}{*}{$G_{2}$} & $\operatorname{FR}\left(M_{f}=35\right)$ & 2.20 & $45.00^{*}$ & 2.00 & 12.40 & 7.15 \\
\hline & $\operatorname{FR}\left(M_{f}=65\right)$ & 2.02 & 65 & 3.50 & 13.00 & 12.8 \\
\hline & $\operatorname{MIGO}\left(M_{s}=2\right)$ & 2 & 46.72 & 2.19 & 9.06 & 5.80 \\
\hline & $\operatorname{MIGO}\left(M_{s}=1.4\right)$ & 1.4 & 67.16 & 3.81 & 11.01 & 10.9 \\
\hline & $\operatorname{AMIGO}\left(M_{s}=2\right)$ & 2.06 & 45.8 & 2.12 & 9.4 & 5.81 \\
\hline & $\operatorname{AMIGO}\left(M_{s}=1.4\right)$ & 1.38 & 70.7 & 3.77 & 12.1 & 12.1 \\
\hline \multirow{6}{*}{$G_{3}$} & $\operatorname{FR}\left(M_{f}=35\right)$ & 1.95 & 35 & 6.03 & 0.01 & 0.01 \\
\hline & $\operatorname{FR}\left(M_{f}=65\right)$ & 1.31 & 65 & 14.7 & 0.057 & 0.057 \\
\hline & $\operatorname{MIGO}\left(M_{s}=2\right)$ & 2 & 35.48 & 4.94 & 0.006 & 0.004 \\
\hline & $\operatorname{MIGO}\left(M_{s}=1.4\right)$ & 1.4 & 51.41 & 12.18 & 0.023 & 0.013 \\
\hline & $\operatorname{AMIGO}\left(M_{s}=2\right)$ & 1.79 & 33.8 & 54.7 & 0.131 & 0.0748 \\
\hline & $\operatorname{AMIGO}\left(M_{s}=1.4\right)$ & 1.28 & 55.4 & 101 & 0.266 & 0.249 \\
\hline \multirow{6}{*}{$G_{4}$} & $\mathrm{FR}\left(M_{f}=35\right)$ & 2.36 & 35 & 2.6 & 1.16 & 1.11 \\
\hline & $\operatorname{FR}\left(M_{f}=65\right)$ & 1.61 & 65 & 4.39 & 2.13 & 2.13 \\
\hline & $\operatorname{MIGO}\left(M_{s}=2\right)$ & 2 & 36.04 & 3.41 & 1.38 & 0.84 \\
\hline & $\operatorname{MIGO}\left(M_{s}=1.4\right)$ & 1.4 & 56.26 & 6.89 & 2.47 & 1.95 \\
\hline & $\operatorname{AMIGO}\left(M_{s}=2\right)$ & 2.26 & 31.2 & 3.64 & 2.62 & 1.24 \\
\hline & $\operatorname{AMIGO}\left(M_{s}=1.4\right)$ & 1.42 & 63.2 & 7 & 3.21 & 3.1 \\
\hline
\end{tabular}

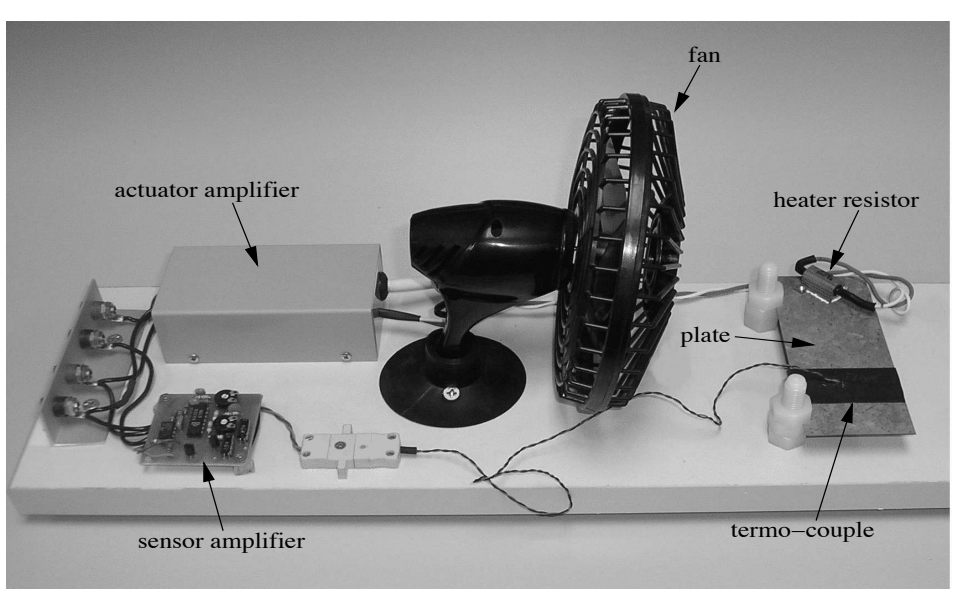

Figure 11. Picture of the thermal process used for the experiment.

response), PID controller design (by applying the proposed method), implementation and closed loop testing and analysis of the results.

\subsection{System identification}

In order to obtain the necessary data to estimate the frequency response of the process, a linear chirp signal is applied to the input of the system, starting from a steady state $\left(u_{e q}=4.5 \mathrm{~V}\right.$, $y_{e q} \approx 67^{\circ}$ ). The input and output signals are presented in the figure 12 . 
Table 4. Comparison of the results reached by the proposed PID design method and the ones in (Panagopoulos et al. 2002) and (Astrom and Hagglund 2004). FR: Frequency response based method, MIGO: M-constrained integral gain optimization method, AMIGO: approximate MIGO method.

\begin{tabular}{|c|c|c|c|c|c|c|}
\hline$G_{n}$ & Design & $M_{s}$ & $\phi_{m}$ & $\gamma_{m}$ & $I A E$ & $I E$ \\
\hline \multirow{6}{*}{$G_{5}$} & $\operatorname{FR}\left(M_{f}=35\right)$ & 2.57 & 35 & 2.00 & 2.78 & 1.88 \\
\hline & $\operatorname{FR}\left(M_{f}=65\right)$ & 1.85 & 65 & 3.50 & 3.45 & 3.45 \\
\hline & $\operatorname{MIGO}\left(M_{s}=2\right)$ & 2 & 37.60 & 2.67 & 2.84 & 1.59 \\
\hline & $\operatorname{MIGO}\left(M_{s}=1.4\right)$ & 1.4 & 59.16 & 5.05 & 4.06 & 3.41 \\
\hline & $\operatorname{AMIGO}\left(M_{s}=2\right)$ & 2.36 & 32.2 & 2.47 & 3.75 & 1.8 \\
\hline & $\operatorname{AMIGO}\left(M_{s}=1.4\right)$ & 1.44 & 66 & 4.74 & 4.42 & 4.42 \\
\hline \multirow{6}{*}{$G_{6}$} & $\operatorname{FR}\left(M_{f}=35\right)$ & 2.53 & 35 & 2.00 & 6.09 & 3.03 \\
\hline & $\operatorname{FR}\left(M_{f}=65\right)$ & 2.09 & 65 & $3.50^{*}$ & 5.25 & 5.25 \\
\hline & $\operatorname{MIGO}\left(M_{s}=2\right)$ & 2 & 39.14 & 2.46 & 4.25 & 2.38 \\
\hline & $\operatorname{MIGO}\left(M_{s}=1.4\right)$ & 1.4 & 61.16 & 4.51 & 5.59 & 4.92 \\
\hline & $\operatorname{AMIGO}\left(M_{s}=2\right)$ & 2.34 & 33.7 & 2.28 & 5.32 & 2.51 \\
\hline & $\operatorname{AMIGO}\left(M_{s}=1.4\right)$ & 1.43 & 65.9 & 4.33 & 5.95 & 5.89 \\
\hline \multirow{6}{*}{$G_{7}$} & $\overline{F R}\left(M_{f}=35\right)$ & 2.37 & 35 & 2.55 & 10.6 & 4.64 \\
\hline & $\operatorname{FR}\left(M_{f}=65\right)$ & 2.18 & 65 & 3.50 & 7.06 & 7.06 \\
\hline & $\operatorname{MIGO}\left(M_{s}=2\right)$ & 2 & 40.56 & 2.37 & 5.61 & 3.20 \\
\hline & $\operatorname{MIGO}\left(M_{s}=1.4\right)$ & 1.4 & 62.77 & 4.26 & 7.38 & 6.44 \\
\hline & $\operatorname{AMIGO}\left(M_{s}=2\right)$ & 2.33 & 35.5 & 2.16 & 6.57 & 3.22 \\
\hline & $\operatorname{AMIGO}\left(M_{s}=1.4\right)$ & 1.43 & 67.1 & 4.04 & 7.43 & 7.4 \\
\hline \multirow{6}{*}{$G_{8}$} & $\mathrm{FR}\left(M_{f}=35\right)$ & 2.25 & $45.00^{*}$ & 2.00 & 6.92 & 4.01 \\
\hline & $\operatorname{FR}\left(M_{f}=65\right)$ & 2.06 & 65 & 3.50 & 8.24 & 7.11 \\
\hline & $\operatorname{MIGO}\left(M_{s}=2\right)$ & 2 & 47.36 & 2.21 & 6.16 & 3.82 \\
\hline & $\operatorname{MIGO}\left(M_{s}=1.4\right)$ & 1.4 & 68.23 & 3.85 & 8.39 & 7.22 \\
\hline & $\operatorname{AMIGO}\left(M_{s}=2\right)$ & 3.22 & 26.3 & 1.49 & 6.65 & 2.35 \\
\hline & $\operatorname{AMIGO}\left(M_{s}=1.4\right)$ & 1.67 & 61.7 & 2.59 & 6.51 & 5.24 \\
\hline
\end{tabular}
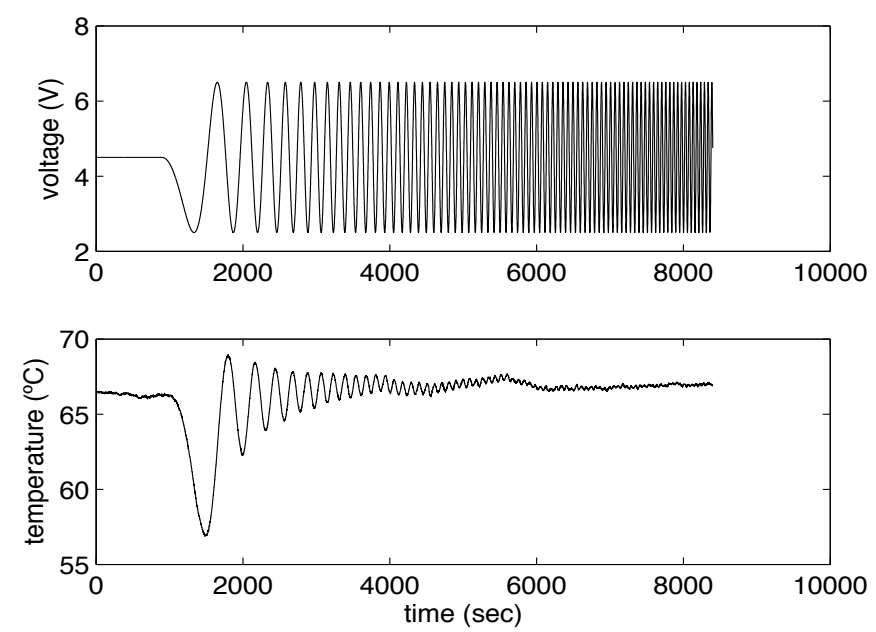

Figure 12. Experimental data used for the estimation of the frequency response. Input and output signals.

The frequency response of the system is estimated from this data set (after subtracting the initial values) by means of spectral analysis. Specifically, a smoothed version of the empirical transfer function estimate was used, (Ljung 1999) (function etfe from Matlab). The corresponding frequency response is displayed in the figure 13. This frequency response represents the non parametric model used for the PID design. 

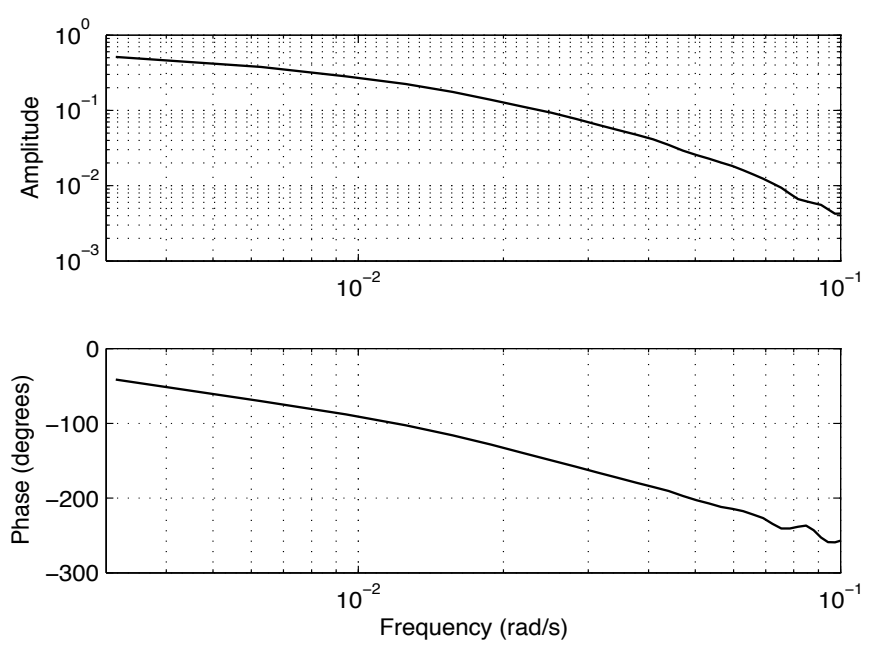

Figure 13. Estimated frequency response of the thermal process obtained applying ETFE on the experimental data.

\subsection{Controller parameters calculation}

The proposed method has been applied to design PID controllers using the previously obtained frequency response. The closed loop system has to satisfy the following requirements: $\phi_{m}=60^{\circ}$, $\gamma_{m}>2$. The filter factor is selected as $N=10$, as the measurement noise is intermediate. The controller gain $K_{i}$ and gain margin are plotted as a function of $a$ in the figure 14. The value of parameter $a$ that leads to a maximum controller gain $K_{i}$ is in this case $a=1.56$ (design A), with a gain margin $\gamma_{m}=3.9$. Two other controllers have been calculated for comparison, using higher and lower $a$ values ( $a=1$, design $\mathrm{B}$ and $a=2.5$, design $\mathrm{C}$ ). The resulting controller parameters are presented in the table 5 .

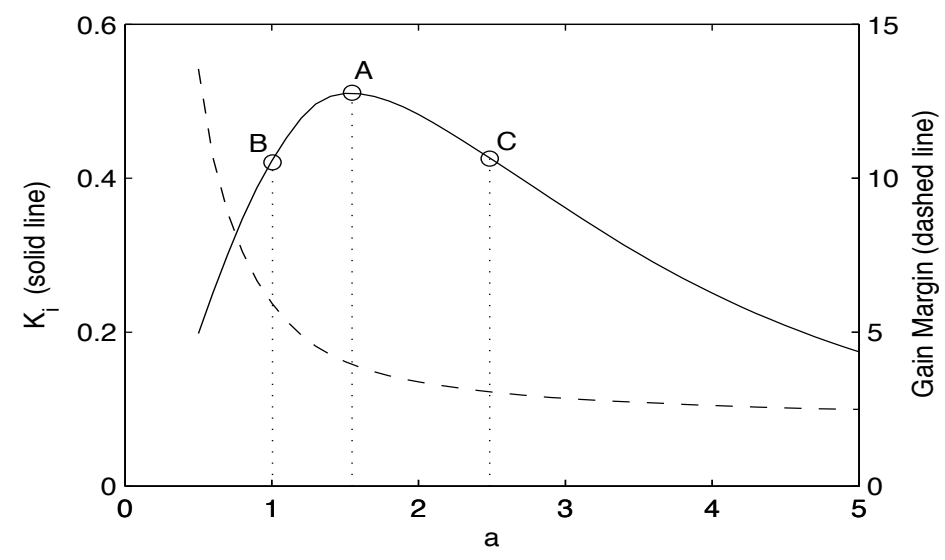

Figure 14. Controller gain $K_{i}$ and Gain Margin as a function of $a$ for the PID controller of the thermal plant

\subsection{Analysis and comparison of the controllers}

The objective of the last part of the experiment has been to analyze and compare the behavior of the three PID controllers. The experimental closed loop response to a step change in reference and to the disturbance produced by the attached fan are displayed for the three designs in the figure 15 . 
The results demonstrates the applicability of the proposed design method to real processes, while shows that the optimum $a$ value (design A) results in the minimum IAE, as shown in table 5 .

Table 5. Parameters of the designed PID controllers and resulting IAE for the thermal plant

\begin{tabular}{cccccc} 
PID & $\mathbf{a}$ & $\mathbf{K p}$ & $\mathbf{T d}$ & $\mathbf{T i}$ & $\mathbf{I A E}$ \\
\hline $\mathbf{A}$ & 1.56 & 7.2 & 31.66 & 141.1 & 409 \\
$\mathbf{B}$ & 1 & 5.27 & 27.93 & 124.47 & 492 \\
$\mathbf{C}$ & 2.5 & 7.71 & 40.59 & 180.93 & 456 \\
\hline
\end{tabular}
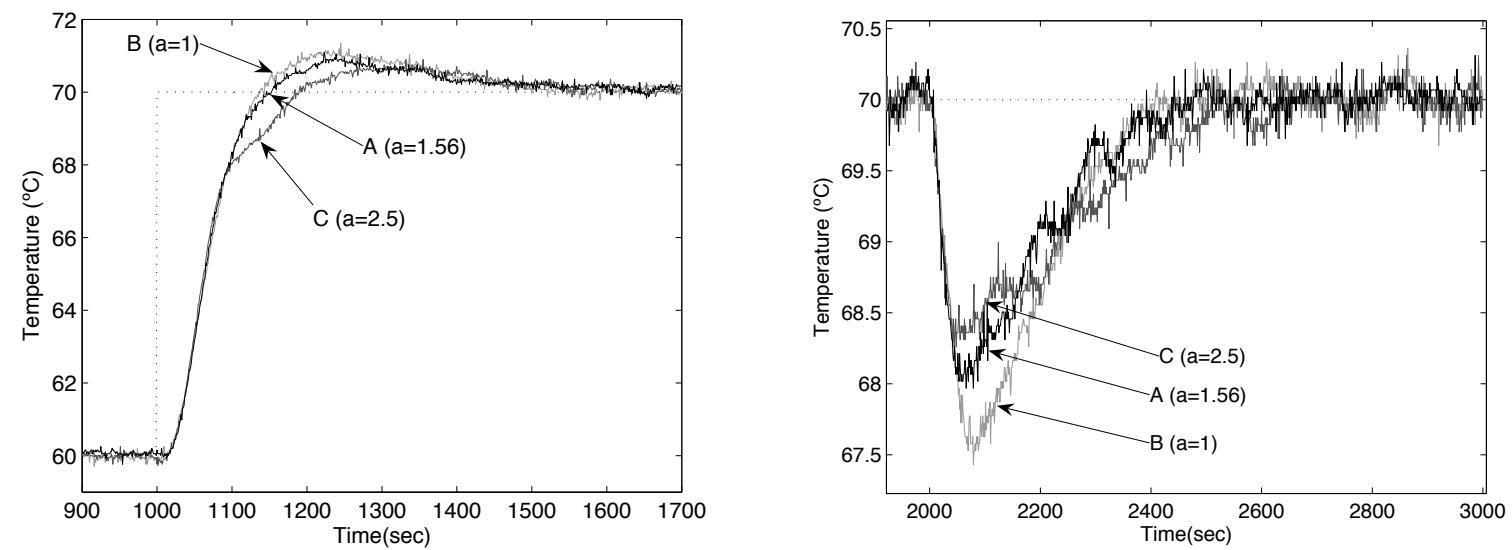

Figure 15. Closed loop response of the thermal system: step reference response (left) and step disturbance response (right).

In order to reduce the overshoot of the step reference response, the proportional term weighting factor $b$ for the reference has been adjusted by trail and error. The figure 16 shows the response with the A controller but using $b=0.7$. For this value of $b$ the overshoot is suppressed.

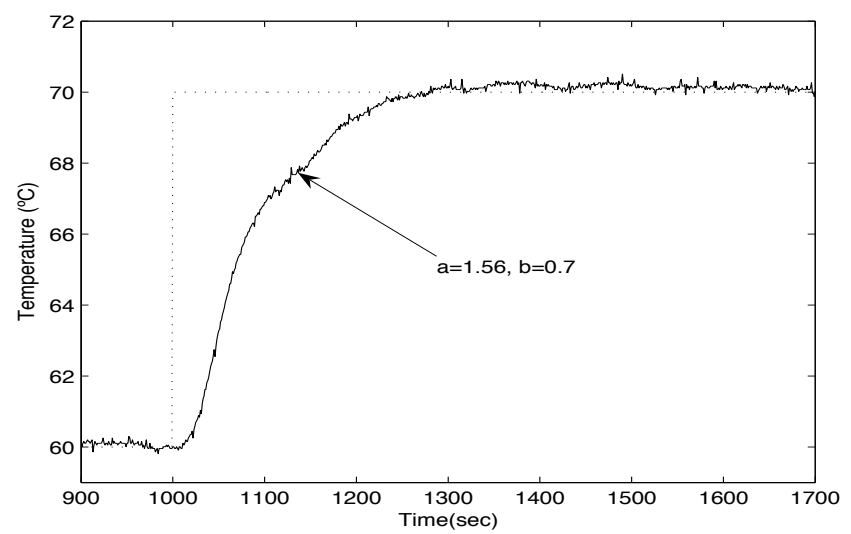

Figure 16. Closed loop response of the thermal plant to step change on reference for the design $\mathrm{A}$ and $\mathrm{b}=0.7$.

\subsection{Straight line approximation}

The chirp signal used for the calculation of the experimental frequency response, shown in figure 12 can be difficult to be applied to industrial processes. In order to overcome this difficulty, and 
to make more applicable in practice the proposed design method, a straight line approximation of the frequency response can be used. For this approximation, only two points of the frequency response are needed. These points can be obtained by a simple relay feedback experiment (as is described for example in (Tan et al. 2005)). The two points should be near the $180^{\circ}$ phase, such that an approximation of the straight tangent line of the Bode plot at that phase is obtained. The figure 17 shows the straight line approximation around the $180^{\circ}$ phase of the frequency response of the plant. The figure 18 shows the controller gain $K_{i}$ and gain margin as a function of parameter $a$ for the straight line approximated frequency response, compared with the full one. The approximate optimum is now $a=1.78$, and the controller parameters $K_{p}=6.39, T_{d}=33.21, T_{i}=148$, very similar to the PID designed with the full frequency response. In order to compare the proposed method and the method described in (Astrom and Hagglund 2004) (AMIGO), a first order with time delay approximate model has been obtained from a step response of the plant, leading to a time constant of $T=250$, a time delay of $L=40$ and gain $K=0.86$. A controller with $M_{s}=1.4$ as design objective has been calculated according to (Astrom and Hagglund 2004), using a filter pole with the same $N$ parameter for both controllers. The response to step reference and disturbance of both controllers can be seen in figure 19. Clearly, the controller designed with the AMIGO method produces a more oscillatory (and hence less robust) response than expected by the selected $M_{s}=1.4$. This is due to the modelling error implicit in the first order with time delay model. In fact, the resulting $M_{s}$ and phase margin (measured from the experimental frequency response of the plant) are $M_{s}=1.75, \phi_{m}=44.9^{\circ}$. On the other hand, the performance of the controller designed with the proposed approach is more robust, as expected, with a resulting $M_{s}=1.53$ and $\phi_{m}=65.2^{\circ}$. Comparing the disturbance response, the resulting IAE is almost double in the AMIGO design, due to the conservativeness of the tuning formulas, especially for low $\tau=L /(L+T)$ plants.
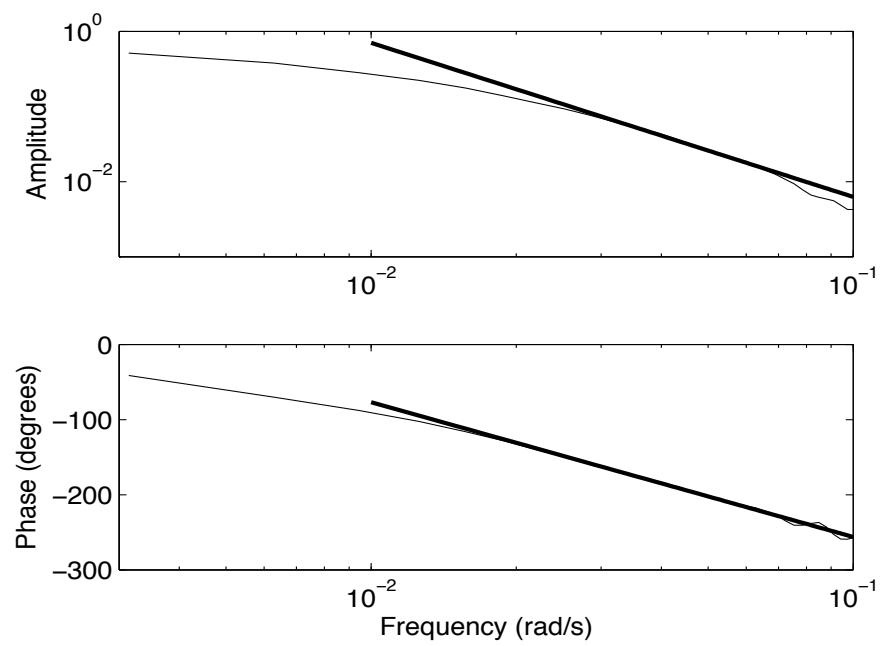

Figure 17. Straight line approximation of the frequency response close to the $180^{\circ}$ phase.

\section{Conclusions}

In this paper, a simple method to design PID controllers in the frequency domain, based on a simplified constrained optimization, has been presented.

The design specifications are assumed to be an exact required phase margin, a minimum required gain margin, and an IE of the disturbance response as low as possible. For PID, the two zeros are imposed to be equal, while the derivative high frequency filter factor $(N)$ is assumed to be previously selected depending on the measurement noise. 


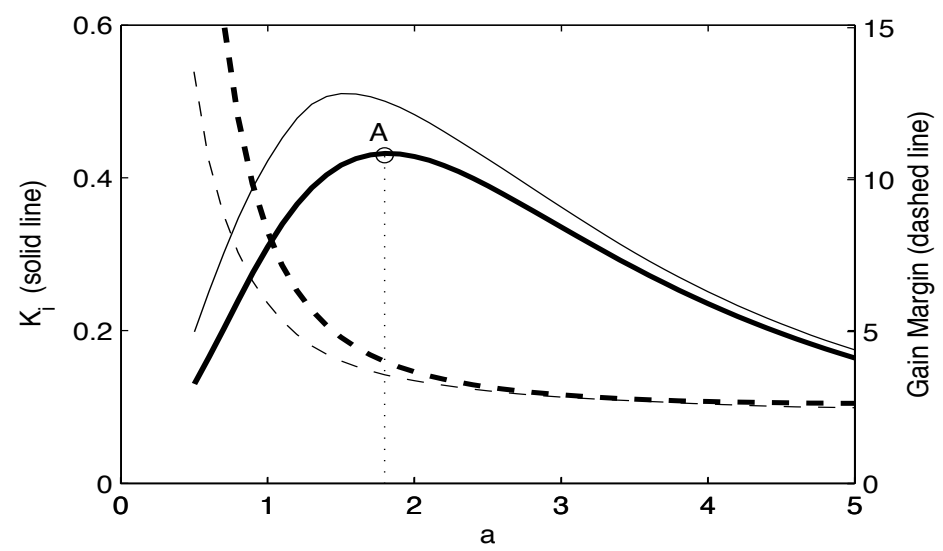

Figure 18. Controller gain $K_{i}$ and Gain Margin as a function of $a$ for PID controller of the thermal plant. Thick line: straight line frequency response. Thin line: full frequency response.

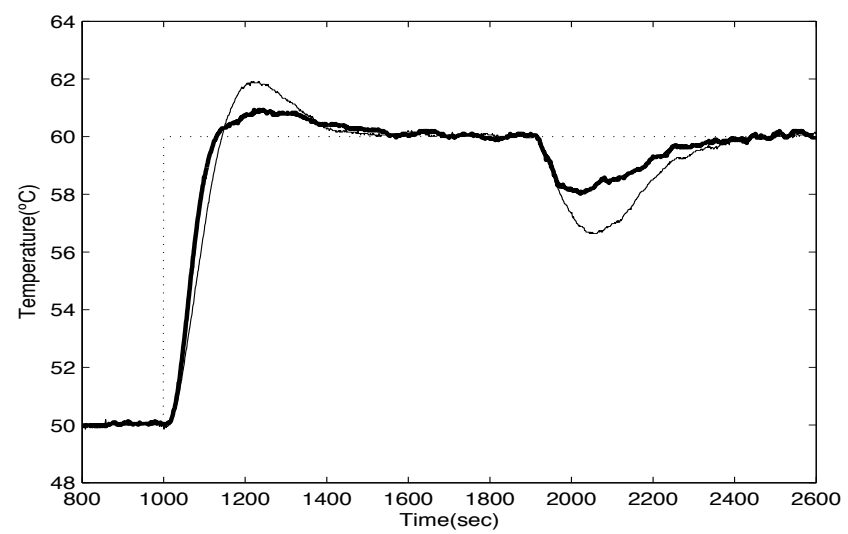

Figure 19. Step reference and disturbance response of thermal plant. Thick line: proposed approach with straight line approximation. Thin line: method of (Astrom and Hagglund 2004).

The design method is based on the use of the quotient between the final crossover frequency and the controller zero as the main tuning parameter.

As an almost optimal tuning strategy for that parameter (in the sense of minimal disturbance IAE or steady state disturbance error), the maximization of the controller gain ( $K_{i}$ for PI and PID, or $K_{p}$ for PD), subject to an equality constraint in the phase margin and an inequality constraint in the gain margin, is proposed. The optimization problem is very easy to solve (can be solved by simple hand calculations), as it only depends on one parameter.

The design method has been tested on a set of systems widely used for test purposes, and compared to other methods. The advantages with respect direct numerical IAE or IE minimization approaches, constrained to a prescribed $M_{s}$ specification, are that the minimization problem is much simpler to solve and the existence of a simple to use tuning parameter to change the design, while the drawbacks are that the performance is slightly worse than the optimal, and that the $M_{s}$ is a better parameter to define the compromise between robustness and performance than the phase and gain margins. The advantage with respect the methods based on a simple model approximation from the step response (first order plus dead time or similar), is that the performance is better, while the drawback is that a more complex experiment is needed to obtain the frequency response data (at least some relay feedback experiments are needed).

The proposed tuning parameter can be easily used to reduce the closed loop bandwidth with respect the optimum one if desired (for example to reduce sensibility to high frequency noise).

For industrial plants where the full frequency response is difficult to be obtained, the proposed approach can be also applied using a simple straight line approximation of the frequency 
response, obtained by simple relay feedback experiments. This idea opens the possibility for designing simple auto tuning methods that can be implemented in low cost embedded computers.

An experimental design case has been developed to demonstrate the practical applicability of the method, including the design based on the straight line approximation of the frequency response.

\section{References}

Astrom, K.J., and Hagglund, T. (2004), "Revisiting the Ziegler-Nichols step response method for PID control," Journal of Process Control, pp. 635-650.

Astrom, K.J., Panagopoulos, H., and Hagglund, T. (1998), "Design of PI Controllers based on Non-Convex Optimization," Automatica, 34, 585-601.

D'azzo, J., and Houpis, C., Feedback Control Systems, Mc Graw Hill (1966).

Dorf, R.C., and Bishop, R., Modern Control Systems, Prentice Hall (2007).

Franklin, G., Powell, J., and Emami-Naeini, A., Feedback Control of Dynamic Systems, Prentice Hall (2005).

Ho, W.K., Lim, K.W., and Wen, X. (1998), "Optimal Gain and Phase Margin Tuning for PID Controllers," Automatica, 34, 1009-1014.

Ho, W.K., Hang, C.C., and Cao, L.S. (1995), "Tuning of PID controllers based on gain and phase margin specifications," Automatica, 31, 497-502.

Hwang, C., and Hsiao, C.Y. (2002), "Solution of a non-convex optimization arising in PI/PID control design," Automatica, 38, 1895-1904.

Krinstianson, B., and Lennartson, B. (2006b), "Evaluation and simple tuning of PID controllers with high-frequency robustness," Journal of Process Control, 26, 91-102.

Krinstianson, B., and Lennartson, B. (2006a), "Robust tuning of PI and PID controllers: using derivative action despite sensor noise," IEEE Control Systems Magazine, 26, 55-69.

Liu, G.P., and Daley, S. (1999), "Optimal-tuning PID controller design in the frequency domain with application to a rotary hydraulic system," Control Engineering Practice,, 7, 821-830.

Ljung, L., System identification. Theory for the user, Prentice Hall (1999).

Madhuranthakam, C.R., Elkamel, A., and Budman, H. (2008), "Optimal tuning of PID controllers for FOPTD, SOPTD and SOPTD with lead processes," Chemical Engineering and Processing: Process Intensification, 47, 251-264.

Ogata, K., Modern Control Engineering, Prentice Hall (2003).

Panagopoulos, H., Astrom, K.J., and Hagglund, T. (2002), "Design of PID controllers based on constrained optimization," IEE Proc.-Control Theory Appl., 149, 32-40.

Phillips, C., and Harbor, R., Feedback Control Systems, Prentice Hall (1999).

Tan, K., Lee, T., and Ferdous, R. (2005), "Automatic PID Controller Tunning-The Nonparametric Approach," PID Control. New identification and design methods, Springer, pp. 147-182.

Tavakoli, S., Griffin, I., and Fleming, P. (2005), "Robust PI Controller for Load Disturbance Rejection and Setpoint Regulation," IEEE International Conference on Control Applications, pp. $1015-1020$.

Toscano, R. (2005), "A simple robust PI/PID controller design via numerical optimization approach," Journal of Process Control, 15, 81-88.

Wang, Q.G., Fung, H.W., and Zhang, Y. (1999), "PID tuning with exact gain and phase margins," ISA Transactions, 39, 243-249. 